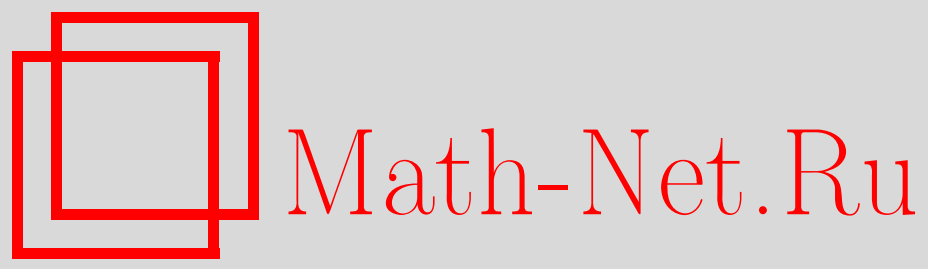

Б. С. Кругликов, Существование близких псевдоголоморфных дисков для почти комплексных многообразий и приложение к псевдонорме КобаясиРойдена, Функи. анализ и его прил., 1999, том 33, выпуск 1, 46-58

DOI: https://doi.org/10.4213/faa337

Использование Общероссийского математического портала MathNet.Ru подразумевает, что вы прочитали и согласны с пользовательским соглашением

http://www.mathnet.ru/rus/agreement

Параметры загрузки:

IP : 18.209 .158 .208

26 апреля 2023 г., 07:22:39

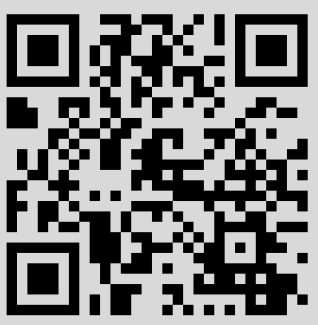




\title{
Существование близких псевдоголоморфных дисков для почти комплексных многообразий и приложение к псевдонорме Кобаяси-Ройдена
}

\author{
(c) 1999. Б. С. Кругликов
}

\section{Введение}

Пусть $\left(M^{2 n}, J\right)$ - почти комплексное многообразие, т.е. $J^{2}=-\mathbb{I} \in$ $T^{*} M \otimes T M$. Отображение $\Phi:\left(M_{1}, J_{1}\right) \rightarrow\left(M_{2}, J_{2}\right)$ называется псевдоголоморфным, если его дифференциал сохраняет комплексное умножение в касательном расслоении: $\Phi_{*} \circ J_{1}=J_{2} \circ \Phi_{*}$.

Обозначим через $e=1 \in T_{0} \mathbb{C}$ единичный вектор. Будем также обозначать через $D_{R}$ диск в $\mathbb{C}$ радиуса $R$, снабженный стандартной комплексной структурой $J_{0}$. Пусть $v \in T_{p} M, p=\tau_{M} v$, где через $\tau_{M}: T M \rightarrow M$ обозначена каноническая проекция. Будем говорить, что в направлении $v$ проходит диск $f: D_{R} \rightarrow M$, если $f_{*} e=v$. В силу теоремы III из [1] существует малый псевдоголоморфный диск в направлении любого вектора $v$. Мы изучаем немалые псевдоголоморфные диски, лежащие в окрестности данного псевдоголоморфного диска. Основным результатом является

ТЕОРема 1. Пусть через точку р на почти комплексном многообразии $\left(M^{2 n}, J\right)$ проходит непостоянныи псевдоголоморфный диск радиуса $R$

$$
f_{0}:\left(D_{R}, J_{0}\right) \rightarrow(M, J), \quad\left(f_{0}\right)_{*}(0) e=v_{0} \neq 0 .
$$

Тогда для любого $\varepsilon>0$ существует такая окрестность $\mathscr{V}=\mathscr{V}_{\varepsilon}\left(v_{0}\right)$ вектора $v_{0} \in T M$, что в направлении каждого вектора $v \in \mathscr{V}$ проходит псевдоголоморфный диск радиуса $R-\varepsilon$

$$
f:\left(D_{R-\varepsilon}, J_{0}\right) \rightarrow(M, J), \quad f_{*}(0) e=v .
$$

Данная теорема имеет важное приложение в теории инвариантных метрик. В 1967 г. Кобаяси [2] ввел инвариантную относительно биголоморфизмов псевдометрику на комплексных многообразиях. Это послужило началом теории гиперболических пространств [3-5]. Псевдометрика Кобаяси это наибольшая псевдометрика среди не увеличивающихся при голоморфных отображениях псевдометрик, которая совпадает на единичном диске $D_{1} \subset \mathbb{C}$ с расстоянием $d_{D}$, индуцированным инфинитезимальной метрикой Пуанкаре модели Лобачевского

$$
d l^{2}=\frac{d z d \bar{z}}{\left(1-|z|^{2}\right)^{2}}
$$

\footnotetext{
* Работа частично поддержана грантом INTAS 96-0713.
} 
На комплексном многообразии $M$ она определяется так:

$$
d_{M}(p, q)=\inf \sum_{k=1}^{m} d_{D}\left(z_{k}, w_{k}\right),
$$

где нижняя грань берется по всем голоморфным отображениям $f_{k}: D_{1} \rightarrow M$, $k=1, \ldots, m$, таким, что $f_{1}\left(z_{1}\right)=p, f_{k}\left(w_{k}\right)=f_{k+1}\left(z_{k+1}\right)$ и $f_{m}\left(w_{m}\right)=q$. В работе [6] псевдометрика Кобаяси была перенесена на случай произвольных почти комплексных многообразий и было показано, что основные свойства этой псевдометрики сохраняются.

В 1970 г. Ройден [7] обнаружил инфинитезимальный аналог этой псевдометрики для комплексных многообразий. Мы определяем соответствующие величины в категории почти комплексных многообразий и с помощью теоремы 1 доказываем теорему о совпадении (теорема 3). Мы доказываем критерий гиперболичности (теорема 4), а также рассматриваем процедуру редукции, позволяющую определить геометрические инварианты пространства модулей псевдоголоморфных кривых.

\section{§1. Существование псевдоголоморфных дисков}

1.1. Переформулировка основного результата. Переформулируем теорему 1 на языке дифференциальных уравнений в подходящих координатах. Вначале выберем эти координаты вдоль диска $f_{0}\left(D_{R}\right) \subset M$. В силу существования изометрических координат на поверхностях [8] диск $f_{0}\left(D_{R}\right)$ может задаваться в локальной комплексной системе координат $\left(z^{1}, \ldots, z^{n}\right)$, определенной в некоторой окрестности этого диска, следующим образом: $\left|z^{1}\right| \leqslant R, z^{2}=\cdots=z^{n}=0$. При этом диск будет псевдоголоморфным, $\left.J\right|_{\operatorname{Im} f_{0}}=J_{0}$, и $v_{0}=(1,0, \ldots, 0) \in T_{0} \mathbb{C} \simeq \mathbb{C}$.

ПРЕДЛОЖЕНИЕ 1. ПодХодящей заменой координат можно добиться, итобиь векторные поля $\partial_{k}=\partial / \partial z^{k}$ u $\bar{\partial}_{k}=\partial / \partial \bar{z}^{k}$ удовлетворяли в точках диска $f_{0}\left(D_{R}\right)$ условиям

$$
J \partial_{k}=i \partial_{k}, \quad J \bar{\partial}_{k}=-i \bar{\partial}_{k} .
$$

ДокАЗАтельство. Для векторных полей $\partial_{1}, \bar{\partial}_{1}$ эти уравнения на диске уже выполнены. Определим далее в точках диска трансверсальные к нему векторные поля $\partial_{k}, \bar{\partial}_{k}, k \geqslant 2$, так, чтобы в каждой точке вся совокупность $2 n$ векторов образовывала базис, а также чтобы выполнялось условие (1). Построив такие поля в точках диска, продолжим их в окрестность с помощью леммы 1 . Полученная структура $J$ совпадает со структурой $J_{0}$ на диске и не обязана совпадать с ней вне диска.

Лемма 1. Пусть вдоль диска $D^{k} \subset \mathbb{R}^{k} \times\{0\}^{n-k} \subset \mathbb{R}^{n}$ заданьи $k$ стандартных коммутируюших касательных полей $v_{i}=\partial_{i}, i=1, \ldots, k, u n-k$ трансверсальньх полей $v_{j}, j=k+1, \ldots, n$, причем в каждой точке $x \in D^{k}$ векторы $v_{1}, \ldots, v_{n}$ образуют базис. Тогда в малой окрестности диска $D^{k}$ существуют координать $x^{i}$, такие, что $v_{i}(x)=\partial_{i}=\partial / \partial x^{i}, i=1, \ldots, n$, для всех $x \in D^{k}$. 
ДокАЗАТЕльство. Поскольку коммутаторы векторных полей вдоль $D^{k}$ определяются их 1-продолжениями вне $D^{k}$, то запишем обшую формулу для 1-продолжения векторного поля $v_{1}$ :

$$
v_{1}=\sum_{r=1}^{n}\left(\delta_{1}^{r}+\sum_{s=k+1}^{n} x^{s} \phi_{s}^{r}\left(x_{1}, \ldots, x_{k}\right)\right) \partial_{r} \bmod \mu^{2} \mathscr{D}
$$

где $\mu^{2} \mathscr{D}$ - подмодуль модуля векторных полей, имеющих на подмногообразии $D^{k} \subset \mathbb{R}^{n}$ нуль второго порядка. Если на диске $D^{k}$

$$
v_{j}=\sum_{s=1}^{n} a_{j}^{s}\left(x_{1}, \ldots, x_{k}\right) \partial_{s}, \quad j=k+1, \ldots, n,
$$

то уравнения $\left[v_{1}, v_{j}\right]=0$ при $x^{k+1}=\cdots=x^{n}$ имеют вид

$$
\sum_{r=1}^{n}\left(\partial_{1} a_{j}^{r}\left(x^{1}, \ldots, x^{k}\right)\right) \partial_{r}=\sum_{s=k+1}^{n} a_{j}^{s}\left(x^{1}, \ldots, x^{k}\right) \sum_{r=1}^{n} \phi_{s}^{r}\left(x^{1}, \ldots, x^{k}\right) \partial_{r} .
$$

Данная система распадается (по $r$ ) на $n$ определенных систем из $n-k$ линейных уравнений с $n-k$ неизвестными. Матрица $\left(a_{j}^{s}\right)_{k+1 \leqslant j, s \leqslant n}$ каждой системы невырожденна, а потому система имеет решение.

Итак, поле $v_{1}$ построено. Выпрямим его: $v_{1}=\partial / \partial x^{1}$. Можно считать, что на диске $D^{k} \subset\left\{x^{k+1}=\cdots=x^{n}=0\right\}$ касательные векторные поля имеют первоначальный вид $v_{i}=\partial_{i}$. В новых координатах коэффициенты разложения (3) уже не зависят от $x^{1}$. Поэтому можно искать продолжение поля $v_{2}$ в виде, аналогичном (2), но без зависимости от $x^{1}$. Продолжая этот процесс, получим координаты $x^{1}, \ldots, x^{n}$, в которых диск $D^{k}$ принадлежит подпространству $\left\{x^{k+1}=\cdots=x^{n}=0\right\}$ и на этом диске

$$
v_{i}=\frac{\partial}{\partial x^{i}}, \quad i=1, \ldots, k, \quad v_{j}=\sum_{s=1}^{n} a_{j}^{s} \partial_{s}, a_{j}^{s}=\mathrm{const}, \quad j=k+1, \ldots, n .
$$

Теперь продолжаем поля в окрестность по формуле (4).

Итак, мы ввели комплексные координаты $z^{k}=x^{k}+i y^{k}$ в окрестности диска $f_{0}\left(D^{k}\right)$. Наше многообразие, будучи суженным, имеет вид

$$
M_{0}=\left\{\left|z_{1}\right| \leqslant R,\left|z_{k}\right| \leqslant R_{1}, k \geqslant 2\right\} \simeq D_{R} \times\left(D_{R_{1}}\right)^{n-1} \subset \mathbb{C}^{n}, \quad R_{1} \ll R,
$$

и структура $J$ имеет в точках диска $D_{R}=\left\{\left(x_{1}, y_{1}, 0, \ldots, 0,0\right)\right\}$ вид

$$
J \frac{\partial}{\partial x^{k}}=\frac{\partial}{\partial y^{k}}, \quad J \frac{\partial}{\partial y^{k}}=-\frac{\partial}{\partial x^{k}} .
$$

Записав уравнение Коши-Римана $f_{*} \circ J_{0}=J \circ f_{*}$ для отображения диска $f:\left(D_{R-\varepsilon}, J_{0}\right) \rightarrow(M, J)$ (аналогично п. 3.3 из [1]) и использовав условия выпрямления (6), мы получим эквивалентную формулировку основного утверждения $\left(\partial, \bar{\partial}\right.$ рассматриваются в координатах $\left.z^{k}\right)$ :

Теорема $1^{\prime}$. Пусть на многообразии $M_{0}$ вида (5) задань $n^{2}$ функций $a_{\bar{m}}^{i}(i, m=1, \ldots, n)$ класса $C^{k+\lambda}, k \in \mathbb{Z}_{+}, \lambda \in(0,1)$. Пусть $\varepsilon \in(0, R)$ 
- произвольное сколь угодно малое действительное число. Если $a_{\bar{m}}^{i}(z)=0$ для всех точек $z \in D_{R} \times\{0\}^{n-1} \subset M_{0}$, то уравнение

$$
\bar{\partial} z^{i}+\sum_{m=1}^{n} a_{\bar{m}}^{i}(z) \bar{\partial} \bar{z}^{m}=0, \quad z^{i}(0)=p^{i}, \quad \partial z^{i}(0)=u^{i}, \quad i=1, \ldots, n,
$$

имеет решение $z^{i}=z^{i}(\zeta) \in C^{k+1+\lambda}\left(D_{R-\varepsilon} ; M_{0}\right)$ nри условии, что окрестность $\mathscr{V}=\mathscr{V}\left(v_{0}\right) \ni v$ вектора $v_{0}=(1,0, \ldots, 0) \in T_{0} M_{0}$ вьгбрана достаточно малой. Здесь $v=(p, u), p=\tau_{M} v \in M_{0}, u \in T_{p} M_{0}$.

\section{2. Покрытие окрестности дисками и еще одна переформули- ровка.}

Теорема $1^{\prime \prime}$. В формулировке теоремь $1^{\prime}$ можно положить $p=0$. Таким образом, уравнение на искомьй псевдоголоморфный диск принимает в выљранных координатах следующий вид:

$$
\begin{gathered}
\left\{\begin{array}{l}
\bar{\partial} z^{1}=-\sum_{m=1}^{n} a_{\bar{m}}^{1}(z) \bar{\partial} \bar{z}^{m}, \\
\bar{\partial} z^{I}=-\sum_{m=1}^{n} a_{\bar{m}}^{I}(z) \bar{\partial} \bar{z}^{m},
\end{array}\right. \\
z^{1}(0)=0, \quad z^{I}(0)=0, \quad\left(\partial z^{1}(0), \partial z^{I}(0)\right)=\left(u^{1}, \ldots, u^{n}\right),
\end{gathered}
$$

где мультииндекс $I-э т о ~(2, \ldots, n)$.

Следующее утверждение доказывает эквивалентность теорем $1^{\prime}$ и $1^{\prime \prime}$.

ПРЕДЛОЖЕНИЕ 2. Для любого псевдоголоморфного диска $f_{0}: D_{R} \rightarrow M_{0} u$ любого $\varepsilon>0$ малая окрестность образа $f_{0}\left(D_{R-\varepsilon}\right)$ может быть покрыта образами близких псевдоголоморфных дисков $f$ радиусов $R-\delta$, где $\delta<\varepsilon$ : $\mathscr{O}\left(\operatorname{Im} f_{0}\left(D_{R-\varepsilon}\right)\right) \subseteq \bigcup_{f} \operatorname{Im} f\left(D_{R-\delta}\right)$.

ДокАЗАтЕльство. Возмутим почти комплексную структуру $J$ в окрестности диска $f_{0}\left(D_{R}\right)$ так, чтобы на границе она совпала со стандартной интегрируемой структурой $J_{0}$ : для любого $\varepsilon>0$ существует такая почти комплексная структура $\widetilde{J}$, что $\widetilde{J}=J$ в малой окрестности диска $f_{0}\left(D_{R-\varepsilon / 2}\right)=$ $D_{R-\varepsilon / 2} \times\{0\}^{n-1} \subset M_{0}$ и $\widetilde{J}=J_{0}$ в окрестности границы многообразия $\widetilde{M}=D_{R} \times\left(D_{\delta}\right)^{n-1} \subset M_{0} \subset \mathbb{C}^{n}$. Далее, можно считать, что $\widetilde{M} \subset \widetilde{M}=$ $S_{R}^{2} \times\left(S_{2 R}^{2}\right)^{n-1} \simeq\left(S^{2}\right)^{n}$, причем $\widehat{M}$ можно снабдить почти комплексной структурой $\widehat{J}$, совпадающей с $\widetilde{J}$ на $\widetilde{M}$ и являюшейся стандартной интегрируемой комплексной структурой $J_{0}$ на дополнении. Снабдим многообразие $\widehat{M}$ симплектической структурой $\omega=\omega_{0}^{(1)} \oplus \omega_{0}^{(2)} \oplus \cdots \oplus \omega_{0}^{(2)}$, где $\omega_{0}$ - стандартная форма объема, причем $\omega_{0}^{(1)}\left(S_{R}^{2}\right)=\pi R^{2}$, а $\omega_{0}^{(2)}\left(S_{2 R}^{2}\right)=4 \pi R^{2}$. Уменьшая при необходимости размер окрестности диска $f_{0}\left(D_{R-\varepsilon / 2}\right)$, можно считать, что почти комплексная структура $\widehat{J}$ подчинена симплектической структуре $\omega$, т.е. $\omega(\xi, \widehat{J} \xi)>0$ для $\xi \neq 0$.

Обозначим через $A \in H_{2}(\widehat{M} ; \mathbb{Z})$ гомологический класс сферы $S_{R}^{2} \times\{*\}^{n-1}$ $\subset \widehat{M}$. Диск $f_{0}\left(D_{R-\varepsilon / 2}\right)$ может быть продолжен до полной рациональной псев- 
доголоморфной кривой $u_{0}: S^{2} \rightarrow \widehat{M}$, лежащей в классе $A$. Рассмотрим пространство $\mathscr{M}(A, \widehat{J})$ полных псевдоголоморфных кривых $u: S^{2} \rightarrow \widehat{M}$ класса $A$. Поскольку класс $A$ не представим в виде суммы гомологических классов $\sum_{i=1}^{n} A_{i}, n \geqslant 2$, с $\omega\left(A_{i}\right)>0$, то из теоремы компактности Громова $([9$, Sec. 1.5.В] или [10, Sec. 4.3.2]) следует компактность пространства $\mathscr{M}(A, \widehat{J}) / G$, где $G \simeq P S L_{2}$ является группой комплексных автоморфизмов сферы $\left(S^{2}, J_{0}\right), \operatorname{dim} G=6$. Кроме того, для почти комплексной структуры $\widehat{J}$ общего вида пространство $\mathscr{M}(A, \widehat{J})$ является гладким многообразием размерности $2 n+4$ [9, Sec. 2.1-2.2; 10, Sec. 3.1.2]. Рассмотрим непараметризованное пространство псевдоголоморфных кривых $\mathscr{W}(A, \widehat{J})=\mathscr{M}(A, \widehat{J}) \times_{G} S^{2}$. Это пространство является компактным многообразием размерности $2 n$. Рассмотрим отображение вычисления значения $e: \mathscr{W}(A, \widehat{J}) \rightarrow \widehat{M}$, действующее по формуле $e(u, z)=u(z)$ для $z \in S^{2}, u \in \mathscr{M}(A, \widehat{J})$. Мы считаем, что группа $G$ действует на $\mathscr{M} \times S^{2}$ сопряжением, $\phi(u, z)=\left(u \circ \phi^{-1}, \phi(z)\right), \phi \in G$, а потому определение отображения $e$ корректно. Так как вне малой окрестности образа $u_{0}\left(S^{2}\right) A$-кривые расслаивают многообразие $\widehat{M}$ (поскольку там $\left.\widehat{J}=J_{0}\right)$, отображение $e$ имеет степень $1, \operatorname{deg} e=1$. Поэтому через любую точку, близкую к кривой $u_{0}\left(S^{2}\right)$, проходит псевдоголоморфная кривая $u\left(S^{2}\right)$, гомологичная кривой $u_{0}\left(S^{2}\right)$.

Чтобы избавиться от условия общности положения для $\widehat{J}$, нужно взять последовательность $\widehat{J}_{k}$ структур общего положения, сходящуюся к $\widehat{J}$ в $C^{\infty}$-топологии, и воспользоваться теоремой компактности $[10, \mathrm{~B} .4 .2]$. Пересекая полученный набор псевдоголоморфных сфер $u\left(S^{2}\right)$ с малой окрестностью $\mathscr{O}$ диска $f\left(D_{R-\varepsilon / 2}\right)$, мы получим искомый набор дисков $f(D)$ в окрестности $(\mathscr{O}, J)$. Гладкость этих дисков вытекает из стандартной эллиптической регулярности [1, Sec. 5.4, 4.3; 10, Sec. B.4.1].

1.3. Пространства, нормы и оценки. Определим $\lambda$-гёльдерову норму комплекснозначных функций на диске $D_{R}$ радиуса $R$ формулой $\|f\|=|f|+$ $(2 R)^{\lambda} H_{\lambda}[f], \lambda \in(0,1)$, где

$$
H_{\lambda}[f]=\sup _{w \neq 0}\left|\frac{f(z+w)-f(z)}{w^{\lambda}}\right|, \quad|f|=\sup |f(z)| .
$$

Пространство $C^{\lambda}\left(D_{R}, M_{0}\right) \quad \lambda$-гёльдеровых отображений состоит из всех отображений $f: D_{R} \rightarrow M_{0}$, компоненты которых имеют конечную $\lambda$-норму, $\left\|f^{i}\right\|<\infty$. Пространство $C^{k+\lambda}\left(D_{R}, M_{0}\right), k \in \mathbb{Z}_{+},(k+\lambda)$-гёльдеровых отображений состоит из отображений, все частные производные которых до порядка $k$ включительно принадлежат $C^{\lambda}$.

Введем также пространство $B=C_{\bullet}^{k+\lambda}\left(D_{R}, M_{0}\right)$, состоящее из отображений $f \in C^{k+\lambda}, f(0)=0$, с нормой $\|f\|=\sum_{1}^{n}\left\|f^{i}\right\|$. Заметим, что пространство $B$ может быть также снабжено нормой

$$
\|f\|^{\prime}=\max \{\|\partial f\|,\|\bar{\partial} f\|\} \text {. }
$$

ПРЕДЛОЖЕНИЕ 3. Пространства $\left(C^{\lambda},\|\cdot\|\right) u\left(C_{\bullet}^{1+\lambda},\|\cdot\|^{\prime}\right)$ банаховы. 
Второе утверждение следует из первого и оценки [1, Sec. 7.1.c-7.1.e]

$$
\|f\| \leqslant 6 R\|f\|^{\prime} .
$$

Рассмотрим операторы Коши

$$
S f(w)=\frac{1}{2 \pi i} \oint_{\partial D_{R}} \frac{f(\zeta)}{\zeta-w} d \zeta, \quad T f(w)=\frac{1}{2 \pi i} \iint_{D_{R}} \frac{f(\zeta)}{\zeta-w} d \zeta \wedge d \bar{\zeta} .
$$

Напомним известные свойства этих операторов [1, Sec. 6.1-6.2]:

$$
\begin{gathered}
f \in C^{\lambda}(D) \Longrightarrow T f \in C^{1+\lambda}(D), \quad \bar{\partial} T f=f, \\
f \in C^{\lambda}(D) \Longrightarrow S f \in C^{\lambda}(\operatorname{Int} D), \quad \bar{\partial} S f=0, \quad S T f=0, \\
f=S f+T \bar{\partial} f \quad(\text { формула Коши-Грина-Помпейю), } \\
\|T f\|^{\prime} \leqslant c_{1}\|f\|, \quad\|S f\| \leqslant c_{2}\|f\| .
\end{gathered}
$$

Рассмотрим также операторы $T_{k} f(w)=T f(w)-\sum_{s=0}^{k} \frac{1}{s !} \partial^{s} T f(0) w^{s}$.

ЛЕмма 2. Для точек $w \in \operatorname{Int} D$ имеет место формула

$$
T_{k} f(w)=\frac{w^{k+1}}{2 \pi i} \iint_{D_{R}} \frac{f(\zeta)}{(\zeta-w) \zeta^{k+1}} d \zeta \wedge d \bar{\zeta}
$$

Лемма 3. Onератор $T_{\infty}=\lim _{k \rightarrow \infty} T_{k}$ определен для функиий $f \in C^{\lambda}\left(D_{R}\right)$, причем $T_{\infty} f \in C^{1+\lambda}\left(D_{R-\varepsilon}\right)$.

ЛЕмма 4. $T_{k}\left(w^{l} \bar{w}^{m}\right)=w^{l} \bar{w}^{m+1} /(m+1)$ npu $l<k+m+2 u T_{k}\left(w^{l} \bar{w}^{m}\right)=$ $w^{l} \bar{w}^{m+1} /(m+1)-R^{2(m+1)} w^{l-m-1} /(m+1) n p u l \geqslant k+m+2$.

СЛЕДСТВИЕ. $T_{\infty}\left(w^{l} \bar{w}^{m}\right)=w^{l} \bar{w}^{m+1} /(m+1)$.

Таким образом, оператор $T_{\infty}$ реализует интегрирование по $\zeta$ на многочленах на $D_{R}$. Наряду с пространством $B$ рассмотрим также его замкнутое подмножество $B_{\delta}=\left\{f=\left(f_{1}, \ldots, f_{n}\right) \in B,\left|f_{1}-\zeta\right| \leqslant \delta,\left|f_{k}\right| \leqslant \delta, k \geqslant 2\right\}$. Мы будем искать решение $f$ уравнения Коши-Римана $(7)$ в пространстве $B_{\delta}$ для малой окрестности $\mathscr{V}$ вектора $v_{0}$.

\section{4. Доказательство теоремы $\mathbf{1}^{\prime \prime}$.}

ИдЕЯ ДОКАЗАТЕЛЬСТВА. В работе [1, Theorem III] было найдено решение уравнения (7) с фиксированным вектором скорости $v$ и малым радиусом диска $R \ll 1$. Для этого рассматривалась линеаризация почти комплексной структуры в точке. В силу близости уравнений на псевдоголоморфные кривые для заданной почти комплексной структуры $J$ и для линеаризованной $J_{0}$ следующее отображение являлось сжимающим:

$$
\Phi: B \rightarrow B, \quad(\Phi f)^{i}(\zeta)=v^{i} \zeta+T_{1}\left(-\sum_{m} a_{\bar{m}}^{i}(f) \bar{\partial} \bar{f}^{m}\right)(\zeta)
$$

В нашем случае радиус диска не является малым; поэтому дословное перенесение аргументов из [1] возможно, только если структура $J$ отличается от $J_{0}$ на диске $f_{0}$ на величину второго порядка малости, т. е. если функции $a_{\overline{1}}^{i}$ 
на диске $f_{0}$ вырождаются вместе со своими производными. В общей ситуации это не так, и мы линеаризуем почти комплексную структуру $J$ вдоль диска $f_{0}$. При этом линеаризация параметризована координатой $z^{1}=\zeta$ на этом диске. Решение комплексного линейного уравнения ведет себя аналогично решению действительного уравнения $\dot{x}=A x+B$ : для немалых значений параметра неверно, что $e^{A t} \approx 1$, и члены ряда $e^{A t}=\sum_{s=0}^{\infty}(A t)^{s} / s$ ! не являются убывающими по модулю; однако это свойство выполняется, начиная с некоторого номера $s \geqslant s_{0}$. Итак, конечные суммы ряда для экспоненты не представляют собой сжимающуюся последовательность, но нужно рассматривать эти суммы, начиная с некоторого члена.

Перейдем к доказательству. Как и в [1], исходя из формул (11), (10), мы ищем решение уравнения (7) в виде (13), но заменяем пространство $B$ на $B_{\delta}$. На самом деле, как указано выше, мы должны изменить определение оператора $\Phi$ для улучшения сходимости. Рассмотрим автоморфизм пространства $\mathbb{C}^{n}$, сжимающий пространство $M_{0}$,

$$
z^{1} \rightarrow z^{1}, \quad z^{I} \rightarrow z^{I} / N, \quad N \gg 1 .
$$

Поскольку $a_{\bar{m}}^{i}=0$ вдоль диска $D_{R} \times\{0\}^{n-1} \subset M_{0}$, то при большом $N$ в уравнении (7) функция $a_{\overline{1}}^{1}$ станет малой, а функции $a_{\overline{1}}^{I}$ будут мало отличаться от своих линеаризаций по переменным $z^{I}$ в норме $\|\cdot\|^{\prime}$.

Следовательно, первое из уравнений (7), рассматриваемое как одно $z^{I}$-параметрическое уравнение, решается методом итераций с применением формулы (13) для комплексной размерности 1 и с заменой $B$ на $B_{\delta}$. При малом $\delta$ и большом $N$ в (14) оценки из [1, Sec. 5.2] доказывают сжимаемость в норме \| • |l' итерационной процедуры, которую мы будем обозначать следующим образом: $z^{1} \mapsto \Psi^{1}\left(z^{1}, z^{I}\right)$.

Для рассмотрения второго из уравнений (7) линеаризуем входящие в него функции по $z^{I}$ :

$$
a_{\overline{1}}^{I}(z)=\sum_{m \geqslant 2}\left(a_{\overline{1} ; m}^{I}\left(z^{1}\right) z^{m}+a_{\overline{1} ; \bar{m}}^{I}\left(z^{1}\right) \bar{z}^{m}\right)+\hat{a}_{\overline{1}}^{I}(z) .
$$

В этой формуле функции $\hat{a}_{\overline{1}}^{I}(z)$ имеют второй порядок малости вдоль диска $D_{R} \subset M_{0}$. Положим также $\hat{a}_{\bar{m}}^{I}(z)=a_{\bar{m}}^{I}(z)$ при $m \neq 1$.

По теореме Вейерштрасса коэффициенты при линейных по $z^{I}$ членах в (15) приближаются в норме $|\cdot|$ на $D_{R}$ многочленами от $z^{1}, \bar{z}^{1}$ :

$$
\begin{gathered}
a_{\overline{1} ; m}^{I}\left(z^{1}\right)=p_{m}^{I}\left(z^{1}, \bar{z}^{1}\right)+\alpha_{m}^{I}\left(z^{1}\right), \\
a_{\overline{1} ; \bar{m}}^{I}\left(z^{1}\right)=p_{\bar{m}}^{I}\left(z^{1}, \bar{z}^{1}\right)+\alpha_{\bar{m}}^{I}\left(z^{1}\right), \quad\left|\alpha_{m}^{I}\right|,\left|\alpha_{\bar{m}}^{I}\right|<\varepsilon .
\end{gathered}
$$

Пусть $A^{I}\left(\zeta, z^{I}\right)=\sum_{m \geqslant 2}\left(p_{m}^{I}(\zeta, \bar{\zeta}) z^{m}+p_{\bar{m}}^{I}(\zeta, \bar{\zeta}) \bar{z}^{m}\right), A_{\delta}^{I}(\zeta, z)=A^{I}\left(\zeta, z^{I}\right)-$ $A^{I}\left(z^{1}, z^{I}\right)$. Тогда второе из уравнений $(7)$ записывается в форме

$$
\bar{\partial} z^{I}(\zeta)=-A^{I}\left(\zeta, z^{I}\right)+U^{I}(z(\zeta))
$$


где слагаемые остатка $U^{I}=A_{\delta}^{I}+U_{1}^{I}+U_{2}^{I}+U_{3}^{I}$ имеют вид

$$
\begin{gathered}
U_{1}^{I}(z)=A^{I}\left(z^{1}, z^{I}\right)\left(1-\bar{\partial} \bar{z}^{1}\right), \quad U_{2}^{I}(z)=-\sum_{m} \hat{a}_{\bar{m}}^{I} \bar{\partial} \bar{z}^{m} \\
U_{3}^{I}(z)=-\sum_{m \geqslant 2}\left(\alpha_{m}^{I}\left(z^{1}\right) z^{m}+\alpha_{\bar{m}}^{I}\left(z^{1}\right) \bar{z}^{m}\right) \bar{\partial} \bar{z}^{1}
\end{gathered}
$$

Уравнение (16) аппроксимируется следующим уравнением с линейной по $z^{I}$ правой частью и полиномиальными по $\zeta$ коэффициентами:

$$
\bar{\partial} z^{I}(\zeta)=-A^{I}\left(\zeta, z^{I}\right)
$$

Его решение строится как предел итерационной процедуры

$$
z_{(k+1)}^{I}=v^{I} \zeta-T_{\infty}\left[A^{I}\left(\zeta, z_{(k)}^{I}\right)\right] .
$$

В силу следствия из леммы 4 итерация интегрирования с помощью оператора $T_{\infty}$ имеет вид $T_{\infty}^{k}\left(w^{l} \bar{w}^{m}\right)=w^{l} m ! \bar{w}^{m+k} /(m+k) !$, и потому процесс итераций (18) сходится при любом начальном значении $z_{(0)}^{I}$ к решению уравнения (17), причем сходимость экспоненциальная. В частности, начиная с некоторого номера $k$, последовательность $z_{(k)}^{I}$ становится сжимающейся. Более того, существуют такие константы $C$ и $\mu$, зависящие только от почти комплексной структуры $J$ (т. е. от коэффициентов $a_{\bar{m}}^{i}$ ), что для любого $k \geqslant 1$ и многочлена $p(\zeta, \bar{\zeta})$ имеет место неравенство

$$
\left\|T_{\infty}^{k}\left[A^{I}(\zeta, p)\right]\right\|^{\prime} \leqslant C e^{\mu R}\|p\| .
$$

Определим теперь итерационную процедуру для вычисления $z^{I}(\zeta)$. Пусть итерационный член $z_{[r]}^{I}$ уже построен. При этом данный член на основании предыдущего шага равен сумме многочлена $P_{[r]}^{I}(\zeta, \bar{\zeta})$ и функции $\theta_{[r]}^{I}(\zeta) \in$ $C^{1+\lambda}$. Представим последнюю функцию по теореме Вейерштрасса в виде суммы многочлена (по $\zeta, \bar{\zeta})$ и погрешности: $\theta_{[r]}^{I}(\zeta)=Q_{[r]}^{I}(\zeta)+q_{[r]}^{I}(\zeta),\left|q_{[r]}^{I}\right| \leqslant$ $\nu\left|\theta_{[r]}^{I}\right|$. Определим следующий член формулой

$$
z_{[r+1]}^{I}(\zeta)=v^{I} \zeta-T_{\infty}\left[A^{I}\left(P_{[r]}^{I}\right)\right]-T_{\infty}^{k_{r}}\left[A^{I}\left(Q_{[r]}^{I}\right)\right]-T_{1}\left[A^{I}\left(q_{[r]}^{I}\right)\right]+T_{1}\left[U^{I}\left(z_{[r]}\right)\right] .
$$

Здесь $A^{I}=A^{I}(\zeta, \cdot)$ и $k_{r}$ - такое число, что, начиная с номера $k_{r}$, последовательность $T_{\infty}^{k}\left[A^{I}\left(Q_{[r]}^{I}\right)\right]$ сжимается с коэффициентом $\varepsilon_{r}$. При этом (см. (8)) имеют место следующие оценки на дополнительные члены:

$$
\begin{aligned}
& \left\|A_{\delta}^{I}\left(\zeta, z^{\prime}\right)-A_{\delta}^{I}\left(\zeta, z^{\prime \prime}\right)\right\| \leqslant c_{3} \delta\left\|z^{\prime}-z^{\prime \prime}\right\|^{\prime}, \quad\left\|U_{1}^{I}\left(z^{\prime}\right)-U_{1}^{I}\left(z^{\prime \prime}\right)\right\| \leqslant c_{3} \delta\left\|z^{\prime}-z^{\prime \prime}\right\|^{\prime}, \\
& \left\|U_{2}^{I}\left(z^{\prime}\right)-U_{2}^{I}\left(z^{\prime \prime}\right)\right\| \leqslant c_{3} \delta\left\|z^{\prime}-z^{\prime \prime}\right\|^{\prime}, \quad\left\|U_{3}^{I}\left(z^{\prime}\right)-U_{3}^{I}\left(z^{\prime \prime}\right)\right\| \leqslant c_{4} \varepsilon\left\|z^{\prime}-z^{\prime \prime}\right\|^{\prime} .
\end{aligned}
$$

Учитывая неравенство (12), мы заключаем, что при малых $\delta, \varepsilon, \varepsilon_{r}$ и $\nu$ последовательность $z_{[r]}^{I}$ будет сжимающейся: $\left\|z_{[r+1]}^{I}-z_{[r]}^{I}\right\|^{\prime} \leqslant(1-\varkappa)\left\|z_{[r]}^{I}-z_{[r-1]}^{I}\right\|^{\prime}$ для некоторого $\varkappa<1$, не зависящего от $r$. Следовательно, учитывая итерацию с помощью $\Psi^{1}$ для переменной $z^{1}$, мы получаем сходяшуюся в $C^{1+\lambda}$ 
последовательность, предел которой и будет нужным решением. Действительно, положим $z_{[r+1]}^{1}=\Psi^{1}\left(z_{[r]}^{1}, z_{[r]}^{I}\right)$, выбрав в качестве параметра $z^{I}$ итерационный член $z_{[r]}^{I}$. Далее, при определении члена $z_{[r+1]}^{I}$ будем считать, что $z^{1}=z_{[r]}^{1}$. Таким образом, мы получаем последовательность $z_{[r]}$.

В силу приведенных оценок и неравенства (19) члены и предел последовательности $z_{[r]}$ отличаются от ее начального члена $z_{[0]}=v \zeta$ менее, чем в экспоненциальное по $R$ количество раз по сравнению с $\left|v-v_{0}\right|$ в норме $\|\cdot\|^{\prime}$. Следовательно, при малом $\left|v-v_{0}\right| \ll 1$ все члены и предел итерационной последовательности лежат в $B_{\delta}$. Значит, последовательность сходится в $B_{\delta}$. Легко видеть, что предел последовательности $z_{[r]}$ будет решением уравнения (7). При условии гладкости коэффициентов $a_{\bar{m}}^{i} \in C^{k+\lambda}\left(M_{0}\right)$ полученное решение гладкости $C^{1+\lambda}$ будет в действительности иметь больший класс гладкости $C^{k+1+\lambda}$. Это следует из эллиптичности уравнения при применении стандартных методов доказательства регулярности [1, Sec. 5.4, 4.3], [10, Sec. В.4.1]. При $a_{\bar{m}}^{i} \in C^{\infty}\left(M_{0}\right)$ мы получаем гладкое решение уравнения Коши-Римана $z(\zeta) \in C^{\infty}\left(D_{R-\varepsilon} ; M_{0}\right)$.

1.5. Пространства струй и связь с $\boldsymbol{h}$-принципом. Назовем расслоением на псевдоголоморфные диски любое вложение (погружение) $\Phi: D_{R} \times$ $N^{2 n-2} \rightarrow M$, такое, что все отображения $\left.\Phi\right|_{D_{R} \times\{x\}}$ псевдоголоморфны, а образ отображения $\Phi$ покрывает все многообразие $M$. Из конструкции предложения 2 с учетом положительности пересечений в размерности 4 [9, Sec. 2.1. $\mathrm{C}_{2} ; 11$, Sec. 1.1] следует

ПРЕДЛОЖЕНИЕ 4. Пусть $(M, J)$ - четырехмерное почти комплексное многообразие. Для любого вложенного (погруженного) псевдоголоморфного диска $f: D_{R} \rightarrow M u$ любого $\varepsilon>0$ малая окрестность образа $f\left(D_{R-\varepsilon}\right)$ допускает расслоение на псевдоголоморфные диски.

Рассмотрим многообразие псевдоголоморфных струй $\mathscr{J}_{P H}^{1}\left(D_{R} ; M\right)$ отображений $u: D_{R} \rightarrow M$. Его точками являются тройки $(\zeta, z, \Phi)$, где $\zeta \in D_{R}$, $z \in M$, а $\Phi:\left(T_{\zeta} D_{R}, J_{0}\right) \rightarrow\left(T_{z} M, J(z)\right)$ - комплексное линейное отображение. В работе [13] показано, что на многообразии $\mathscr{J}_{P H}^{1}$ сушествует каноническая почти комплексная структура $J_{[1]}$, равная $J_{0} \oplus J \oplus J$ в индуцированном минимальной связностью разложении $T_{p} \mathscr{J}_{P H}^{1}=T_{\zeta} D_{R} \oplus T_{z} M \oplus T_{p} \mathscr{F}$, где $\mathscr{F}$ слой естественной проекции $\tau: \mathscr{J}_{P H}^{1}\left(D_{R}, M\right) \rightarrow D_{R} \times M$. Каноническая проекция $\pi: \mathscr{J}_{P H}^{1}\left(D_{R} ; M\right) \rightarrow M$ является псевдоголоморфным отображением, и любое псевдоголоморфное отображение $f: D_{R} \rightarrow M$ канонически поднимается до псевдоголоморфного отображения $j^{1} f: D_{R} \rightarrow \mathscr{J}_{P H}^{1}\left(D_{R} ; M\right)$, $j^{1} f(\zeta)=\left(\zeta, f(\zeta), d_{\zeta} f\right)$.

Мы определим структуру $J_{[1]}$ другим способом (ср. [14, замечание 1]). Если $p=(\zeta, z, \Phi) \in \mathscr{J}_{P H}^{1}$, то можно считать, что отображение $\Phi$ является дифференциалом в точке $\zeta$ малого псевдоголоморфного диска $u: D_{\varepsilon} \rightarrow M$. Обозначим через $p^{(2)} 2$-струю диска $u$ в точке $\zeta \in D_{\varepsilon} \subset D_{R}$. Рассмотрим отображение $j^{1} u: D_{\varepsilon} \rightarrow \mathscr{J}_{P H}^{1}$. Касательное пространство в точке $p$ зависит только от значения $p^{(2)}$. Обозначим его через $L_{p^{(2)}}$. 
Рассмотрим естественную проекцию $\rho: \mathscr{J}_{P H}^{1} \rightarrow D_{R}$ со слоем $\mathscr{H}$. Имеем $T_{p} \mathscr{J}_{P H}^{1}=L_{p^{(2)}} \oplus T_{p} \mathscr{H}$, причем оба слагаемых естественным образом наделены комплексной структурой. Положим $J_{[1]}=J_{0} \oplus J$. Эта структура не зависит от выбора $p^{(2)}$, т. е. определена канонически.

ОПРЕДЕЛЕНИЕ. Назовем псевдоголоморфный диск $g: D_{R} \rightarrow \mathscr{J}_{P H}^{1}\left(D_{R}, M\right)$ голономным, если отображение $g$ является струйным поднятием псевдоголоморфного диска из $D_{R}$ в $M: g=j^{1} f$.

Из предложения 2 для голономного диска $g=j^{1} f: D_{R} \rightarrow \mathscr{J}_{P H}^{1}$ следует существование псевдоголоморфного диска $g^{\prime}$ через любую сколь угодно близкую к образу диска $g$ точку, не обязанного, однако, быть голономным, $g^{\prime} \neq j^{1}\left(\pi \circ g^{\prime}\right)$. В этом смысле теорема 1 дает более сильное утверждение. Действительно, близость начальных точек дисков $g=j^{1} f$ и $g^{\prime}=j^{1} f^{\prime}$ в $\mathscr{J}_{P H}^{1}\left(D_{R-\varepsilon} ; M\right)$ означает близость начальных точек и направлений отображений $f$ и $f^{\prime}$ в $T M$. Поэтому из теоремы 1 следует существование $C^{1}$-близкого диска $f^{\prime}$, и мы можем положить $g^{\prime}=j^{1} f^{\prime}$. Итак, доказана

ТЕОремА 2. Через любую точку, близкую $\kappa$ образу вложенного (погруженного) голономного псевдоголоморфного диска $g: D_{R} \rightarrow \mathscr{J}_{P H}^{1}\left(D_{R} ; M\right)$ проходит вложенный (погруженный) голономный псевдоголоморфньй диск $g^{\prime}: D_{R-\varepsilon} \rightarrow \mathscr{J}_{P H}^{1}\left(D_{R-\varepsilon} ; M\right)$.

Другими словами, предложение 2 остается верным в голономной ситуации. Доказанное утверждение является частным случаем $h$-принципа [15]. Также представляет интерес голономная версия предложения 4.

\section{§2. Псевдонорма Кобаяси-Ройдена}

2.1. Определение псевдонормы и основные свойства. Рассмотрим множество $\mathscr{R}(v)=\bigcup_{r>0} \mathscr{R}_{r}(v)$, где $\mathscr{R}_{r}(v)$ для $r \in \mathbb{R}_{+}$состоит из псевдоголоморфных отображений $f: D_{1} \rightarrow M$, таких, что $f_{*}(0) e=r v$.

ОПРЕДЕЛЕНИЕ. Псевдонормой Кобаяси-Ройдена на почти комплексном многообразии $M$ назовем функцию на касательном расслоении $T M$, определенную формулой

$$
F_{M}(v)=\inf _{\mathscr{R}(v)} \frac{1}{r} .
$$

По теореме III из [1] множество $\mathscr{R}_{r}(v)$ непусто для малых $r$, а потому определение корректно. Мы называем $F_{M}$ псевдонормой, поскольку эта функция является неотрицательной и однородной степени один: $F_{M}(t v)=|t| F_{M}(v)$. Однако $F_{M}$ может обращаться в нуль на некоторых направлениях, и неравенство треугольника не выполнено. Из определения следует

ПРЕДЛОЖЕНИЕ 5. Для яюбого вектора $v \in T M_{1}$ и для яюбого псевдоголоморфного отображения $f:\left(M_{1}, J_{1}\right) \rightarrow\left(M_{2}, J_{2}\right)$ имеем

$$
F_{M_{2}}\left(f_{*} v\right) \leqslant F_{M_{1}}(v) .
$$

Фиксируем произвольную норму | • | на $T M$. 
ПРЕДЛОЖЕНИЕ 6. (i) Для любого компакта $K \subset M$ существует константа $C_{K}$, такая, что для каждого вектора $v \in T M$ с $\tau_{M} v \in K$

$$
F_{M}(v) \leqslant C_{K}|v|
$$

(ii) Пусть $M$ - компактное многообразие (возможно, с краем) с почти комплексной структурой $J$, подчиненной точной симплектической форме $\omega=d \alpha, \omega(\xi, J \xi)>0$ для $\xi \neq 0$. Тогда существует константа $c_{M}>0$, такая, что для всех $v \in T M$

$$
F_{M}(v) \geqslant c_{M}|v|
$$

ДокАЗАТЕЛЬСтво. Для малой окрестности $U$ точки $p \in M$ из оценок п. 5.2а работы [1] следует существование зависящего только от почти комплексной структуры $J$ и окрестности $U$ числа $\varepsilon>0$, такого, что для любых $q \in U, v \in T_{q} M,|v|=1$, и $r \in(0, \varepsilon)$ существует псевдоголоморфный диск $f: D_{1} \rightarrow M$, такой, что $f(0)=q, f_{*}(0) e=r v$. Полагая $C_{U}=1 / \varepsilon$, мы имеем $F_{M}(v) \leqslant C_{U}|v|$ для всех (уже не обязательно единичных) векторов $v$ с $\tau_{M} v \in U$. Поскольку компактное множество может быть покрыто конечным числом окрестностей $U$, первая часть предложения доказана. Вторая часть является переформулировкой нелинейной леммы Шварца $[9, \mathrm{Sec}$. 1.3.A]: если почти комплексная структура $J$ на компактном многообразии подчинена точной симплектической структуре $\omega$, то производная в нуле любого псевдоголоморфного диска $f: D_{1} \rightarrow M$, проходящего через фиксированную точку многообразия, ограничена константой, не зависящей от выбранного диска: $\left|f_{*}(0) e\right|<C$.

ПРЕДЛОЖЕНИЕ 7. ФунКция $F_{M}$ полунепрерьвна сверху.

ДокАЗАтЕльство. Неравенство $\varlimsup_{\lim } \rightarrow v_{0} F_{M}(v) \leqslant F_{M}\left(v_{0}\right)$ равносильно утверждению теоремы 1 , поскольку $F_{M}(v)=\inf (1 / R)$, где нижняя грань рассматривается для всех отображений $f: D_{R} \rightarrow M$, таких, что $f_{*}(0) e=v$.

2.2. Теорема о совпадении. Определим функцию $\bar{d}_{M}: M \times M \rightarrow \mathbb{R}$ формулой

$$
\bar{d}_{M}(p, q)=\inf _{\gamma} \int_{0}^{1} F_{M}(\dot{\gamma}(t)) d t,
$$

где нижняя грань берется по всем кусочно-гладким путям из точки $p$ в $q$. Из предложений 6(i) и 7 следует корректность данного определения и

ПРЕДЛОЖЕНИЕ 8. Функиия $\bar{d}_{M}$ является псевдометрикой.

ТЕорема 3. Введенная псевдометрика совпадает с псевдометрикой Кобалси, $d_{M}=\bar{d}_{M}$.

ДокАЗАтельство. Так как $F_{M}(v)=\inf |\xi|$, где нижняя грань берется по всем псевдоголоморфным отображениям $f: D_{1} \rightarrow M, f_{*} \xi=v$, а норма - относительно метрики Пуанкаре, то неравенство $\bar{d}_{M} \leqslant d_{M}$ очевидно. Докажем обратное. Мы следуем доказательству Ройдена [7].

Пусть $\gamma$ - гладкая кривая из точки $p$ в точку $q$, такая, что $\int_{\gamma} F_{M}<$ $\bar{d}_{M}(p, q)+\varepsilon$. В силу полунепрерывности сверху существует непрерывная на 
$[0,1]$ функция $h$, такая, что $h(t)>F_{M}(\dot{\gamma}(t))$ и

$$
\int_{0}^{1} h(t) d t<\bar{d}_{M}(p, q)+\varepsilon
$$

т.е. для достаточно плотного разбиения $0=t_{0}<t_{1}<\cdots<t_{k}=1$ имеем

$$
\sum_{i=1}^{k} h\left(t_{i-1}\right)\left(t_{i}-t_{i-1}\right)<\bar{d}_{M}(p, q)+\varepsilon .
$$

Рассмотрим произвольную псевдоголоморфную кривую $u_{t}^{\gamma}: D_{\delta} \rightarrow M$, удовлетворяющую условиям $u_{t}^{\gamma}(0)=\gamma(t)$ и $\left(u_{t}^{\gamma}\right)_{*} e=\dot{\gamma}(t)$. Определим для малых $\Delta t \in \mathbb{R}_{+} \subset \mathbb{C}$ кривую $\hat{\gamma}(t ; \Delta t)=u_{t}^{\gamma}(\Delta t)$. Так как $\hat{\gamma}(t ; \Delta t)=\gamma(t+\Delta t)+$ $O\left(|\Delta t|^{2}\right)$, то в силу предложений 8 и 6 для малых $\Delta t$

$$
\begin{aligned}
d_{M}(\gamma(t), \gamma(t+\Delta t)) & \leqslant d_{M}(\gamma(t), \hat{\gamma}(t ; \Delta t))+d_{M}(\hat{\gamma}(t ; \Delta t), \gamma(t+\Delta t)) \\
& \leqslant F_{M}(\dot{\gamma}(t)) \Delta t+O\left(|\Delta t|^{2}\right) \leqslant(1+\varepsilon) h(t) \Delta t .
\end{aligned}
$$

Таким образом, для достаточно плотного разбиения

$$
d_{M}(p, q) \leqslant \sum_{i=1}^{k} d_{M}\left(\gamma\left(t_{i-1}\right), \gamma\left(t_{i}\right)\right)<(1+\varepsilon)\left(\bar{d}_{M}(p, q)+\varepsilon\right) \text {. }
$$

Так как $\varepsilon>0$ произвольно, то теорема доказана.

\section{3. Гиперболичность и негиперболичность.}

ОПРЕДЕЛЕНИЕ. Почти комплексное многообразие $(M, J)$ называется $2 и$ перболическим, если псевдометрика $d_{M}$ является метрикой.

Рассмотрим расслоение единичных касательных векторов $\tau_{M}^{(1)}: T_{1} M \rightarrow$ $M$ для нормы $|\cdot|$, и пусть $F_{M}^{(1)}: T_{1} M \rightarrow \mathbb{R}$ - ограничение псевдонормы Кобаяси-Ройдена. Из предложения 6(i) и теоремы 3 следует

ТЕОремА 4. Функиия $F_{M}^{(1)}$ является ограниченной на компактных подмножествах в М. Многообразие $M$ является гиперболическим, если и только если $F_{M}^{(1)}$ отделена от нуля на компактных подмножествах.

Предположим теперь, что многообразие $M$ не является гиперболическим, например, допускает псевдоголоморфные сферы. В случае почти комплексной структуры $J$ общего вида, подчиненной некоторой симплектической форме $\omega$ на $M$, множество псевдоголоморфных сфер в заданном гомологическом классе $A \in H_{2}(M ; \mathbb{Z}$ ) (пополненных для компактности множеством разложимых рациональных кривых) является конечномерным многообразием $\mathscr{M}(A ; J)[9,10]$. Мы определим при помощи прочедуры редукиии псевдометрику на этом многообразии. А именно, положим для любых двух псевдоголоморфных сфер $f_{i}: S^{2} \rightarrow M$, определенных с точностью до перепараметризации сферы $S^{2}$,

$$
d_{\mathscr{M}}\left(\left[f_{1}\right],\left[f_{2}\right]\right)=d_{M}\left(p_{1}, p_{2}\right),
$$

где $p_{i} \in \operatorname{Im}\left(f_{i}\right)$ - произвольные точки образов. Легко видеть, что $d_{\mathscr{M}}$ является корректно определенной псевдометрикой на многообразии $\mathscr{M}$. 
Псевдометрика $d_{\mathscr{M}}$ является, например, метрикой для почти комплексного многообразия $M^{4}=\Sigma_{g}^{2} \times S^{2}$ с $g>1$ : как и в предложении 4, доказывается расслоенность $M^{4}$ на псевдоголоморфные сферы и изоморфизм $\mathscr{M} \simeq \Sigma_{g}^{2}$. Однако в случае четырехмерных многообразий данное определение интересно лишь для нулевых самопересечений. Действительно, если $A \cdot A>0$ (в неисключительных случаях $A \cdot A \geqslant 0[11])$, то две сферы $\operatorname{Im}\left(f_{1}\right)$ и $\operatorname{Im}\left(f_{2}\right)$ данного гомологического класса пересекаются. Поэтому $d_{\mathscr{M}}\left(\left[f_{1}\right],\left[f_{2}\right]\right)=0$.

В работе [16] показано, что для больших $N$ многообразие $\mathscr{M} \times \mathbb{R}^{2 N}$ обладает гомотопически канонической почти комплексной структурой $\widetilde{J}$. Псевдометрика Кобаяси $d_{\mathscr{M} \times \mathbb{R}^{2 N}}$ индуцирует псевдометрику $\hat{d}_{\mathscr{M}}$ на $\mathscr{M}$ при редукции по $\mathbb{R}^{2 N}$. Возникает естественный вопрос о существовании таких структур $\widetilde{J}$, что псевдометрики $\hat{d}_{\mathscr{M}}$ и $d_{\mathscr{M}}$ совпадают.

\section{ЛитеРАТУРА}

1. Nijenhuis A., Woolf $W$. Some integration problems in almost-complex and complex manifolds. Ann. Math., 77, 424-489 (1963).

2. Kobayashi $S$. Invariant distances on complex manifolds and holomorphic mappings. J. Math. Soc. Japan, 19, 460-480 (1967).

3. Kobayashi $S$. Intrinsic distances, measures, and geometric function theory. Bull. Amer. Math. Soc., 82, 357-416 (1976).

4. Lang S. Introduction to Complex Hyperbolic Spaces. Springer-Verlag, 1987.

5. Полеикий E. А., Шабат Б. В. Инвариантные метрики. В кн.: Современные проблемы математики, Фундамент. напр., т. 9, ВИНИТИ, М., 1986, с. 73-125.

6. Kruglikov B., Overholt $M$. The Kobayashi pseudodistance on almost complex manifolds, Preprint Univ. Tromsoe, 97-19 (1997); e-print: http://xxx.lanl.gov/abs/dgga/9703005.

7. Royden H. L. Remarks on the Kobayashi metric, In: Lect. Notes in Math., vol. 185, 1971, pp. 125-137.

8. Chern S. S. An elementary proof of the existence of isothermal parameter on a surface. Proc. Amer. Math. Soc., 6, 771-782 (1955).

9. Gromov M. Pseudo-holomorphic curves in symplectic manifolds. Invent. Math., 82, 307-347 (1985).

10. McDuff D., Salamon D. J-holomorphic curves and Quantum cohomology. Amer. Math. Soc., Univ. Lecture Series, vol. 6, 1994.

11. McDuff $D$. The local behaviour of holomorphic curves in almost complex 4-manifolds. J. Differential Geom., 34, 143-164 (1991).

12. $M c D u f f D$. Blow ups and symplectic embeddings in dimension 4. Topology, 30, No. 3, 409-421 (1991).

13. Gauduchon $P$. The canonical almost complex structure on the manifold of 1-jets of pseudo-holomorphic mappings between two almost complex manifolds. In: Holomorphic curves in symplectic geometry (M. Audin, J. Lafontaine, eds.), Progr. in Math., vol. 117, Birkhäuser Verlag, 1994, pp. 69-73.

14. Кругликов Б. С. Тензоры Ниенхейса и препятствия к построению псевдоголоморфных отображений. Матем. заметки, 63, вып. 4, 541-561 (1998).

15. Громов М. Л. Дифференциальные соотношения с частными производными, Мир, М., 1990.

16. McDuff D. Examples of symplectic structures. Invent. Math., 89, 13-36 (1987).

Московский государственный технический 